\title{
Simple method of monitoring colonising microbial load in chronic bronchial sepsis: pilot comparison of reduction in colonising microbial load with antibiotics given intermittently and continuously
}

\author{
D C CURRIE, ELIZABETH HIGGS, SARAH METCALFE, D E ROBERTS, P J COLE
}

From the Host Defence Unit, Department of Thoracic Medicine, Cardiothoracic Institute, Brompton Hospital, London

SUMMARY Aerobic and anaerobic culture of sputum on selective bacteriological media, combined with a new method of plating and plate reading, permitted rapid identification and quantitation of three genera of bacteria commonly associated with chronic bronchial sepsis (Haemophilus spp, Pseudomonas aeruginosa, and Staphylococcus aureus) and avoided time consuming serial dilution of sputum and subculture of organisms.

The accuracy of this new technique was assessed in patients with chronic bronchial sepsis and was used to detect changes in the colonising microbial load of Haemophilus spp and Ps aeruginosa in patients with bronchiectasis receiving one of three different antibiotic regimens: intermittent seven day courses of amoxycillin for exacerbations; or a six month course of continuous oral or nebulised amoxycillin. The colonising microbial load of Haemophilus spp was reduced only temporarily $(+++$ to ++$)$ after each intermittent course of antibiotic, but a sustained and greater reduction in the colonising microbial load of both Haemophilus spp $(+++$ to +$)$ and antibiotic resistant $P$ aeruginosa $(+++$ to +$)$ was seen during both continuous treatments. Sputum purulence decreased in parallel with colonising microbial load, reflecting a reduction in host inflammatory response to the colonising microbial load.

One of the problems in treating patients with chronic bronchial sepsis is lack of a satisfactory means of monitoring the effect of treatment on the pathogenesis of the disease. Such a method of detecting, be it directly or indirectly, the factors entailed in disease progression is required if treatment is to halt progression rather than simply to ameliorate symptoms. Several possible variables have accordingly been proposed, including volume and purulence of sputum, its elastolytic activity, and respiratory function. Unfortunately, these are usually inadequateeither monitoring a result of treatment at some distance from the basic pathogenetic mechanism, measuring only one of a complex series of enzymes in sputum, or reflecting already irreversible changes.

Haemophilus influenzae, Haemophilus parainfluenzae, Pseudomonas aeruginosa, and Staphy-

Accepted for publication 4 March 1987 lococcus aureus are the micro-organisms most commonly isolated in patients with chronic bronchial sepsis; and not only are they often present in the sputum of patients suffering exacerbations of their symptoms, but also during clinical remissions. An hypothesis has been proposed that respiratory damage in patients with chronic bronchial sepsis is caused by the host inflammatory response to such colonising micro-organisms and that the microbes, the tissue damage sustained, and the products of inflammation all impair clearance mechanisms so that a "vicious circle" of microbial colonisation, host response, lung damage, and further microbial colonisation ensues. ${ }^{1}$ Serial measurement of colonising microbial load (CML) in the respiratory tract would therefore monitor a central determinant of the pathogenesis and progression of chronic bronchial sepsis, no matter by which pathway subsequent lung damage may occur.

We describe a simple microbiological method that has been developed to measure the colonising micro- 
bial load of each of the most commonly isolated organisms in the sputum of patients with chronic bronchial sepsis, and propose that this will enable physicians to monitor the natural history and response to treatment of the disease. We describe the application of this technique to determine whether the colonising microbial load is reduced most effectively by continuous oral or nebulised antibiotic treatment, or simply by intermittent treatment of exacerbations.

\section{Material and methods}

The following media were used for isolating various of the micro-organisms:

1 All bacteria: blood agar, consisting of Oxoid blood agar base No 2 (CM271) with 7\% defibrinated horse blood added.

2 Staphylococcus: Oxoid mannitol salt (MS) agar (CM85)

3 Pseudomonas: Oxoid cetrimide and nalidixic acid (CN) agar (CM559 and SR102)

4 Haemophilus: bacitracin (BC) agar, ${ }^{2}$ consisting of $100 \mathrm{ml}$ of Oxoid blood agar base No 2 (CM271), $1 \mathrm{ml}$ bacitracin (Mast), $10 \mathrm{mg} / \mathrm{ml}), 200 \mu \mathrm{l}$ haemin (British Drug Houses, $1 \mathrm{mg} / \mathrm{ml}$ ), $1 \mathrm{ml} \mathrm{N}$-acetyl-Dglucosamine (Sigma, $100 \mathrm{mg} / \mathrm{ml}$ ), and $240 \mu \mathrm{l}$ of $50 \% \mathrm{w} / \mathrm{v}$ glucose. A V-factor disc (6 $\mu \mathrm{g}$, Mast) was placed on the surface of the medium.

Phosphate buffered saline (PBS) was prepared from Oxoid PBS tablets (Dulbecco A).

Each patient studied had radiographically confirmed bronchiectasis, and persistently expectorated purulent sputum daily. Expectorated sputum was collected in universal containers from 7 am to 11 am (morning sputum). The whole specimen was well mixed by a whirlimixer. A $3 \mathrm{ml}$ aliquot was diluted with an equal volume of PBS and mixed until homogeneous. Ten microlitres of the diluted sputum was plated on each culture medium using a sterile inoculating loop (initially held vertical in the sputum to ensure an accurate loop volume). The loopful of diluted sputum was streaked across one half of a Petri dish filled with agar. The same loop was used to streak from the second to the third quarter of the dish. One streak with the same loop took the remaining inoculum to the fourth quarter where it was spread out. The three selective media and the blood agar were inoculated in this manner.

$S$ aureus is the only common organism which will grow on mannitol salt agar and ferment the mannitol when incubated aerobically overnight at $37^{\circ} \mathrm{C}$. Ps aeruginosa grows selectively on cetrimide and nalidixic acid agar when incubated aerobically at $37^{\circ} \mathrm{C}$ overnight. For culture and identification of $H$ influenzae, or $H$ parainfluenzae, $\mathrm{BC}$ agar was used and a V disc was applied to the second quarter of the plate after streaking with sputum. This plate was incubated at $37^{\circ} \mathrm{C}$ overnight, anaerobically to inhibit growth of Ps aeruginosa. ${ }^{2}$ Growth of Haemophilus spp was confined to the area of the $\mathrm{V}$ disc which allowed these colonies to be distinguished from coliforms. In doubtful cases the organisms were subcultured to check their identity. $H$ influenzae was not routinely distinguished from $H$ parainfluenzae. Sputum culture on blood agar permitted qualitative assessment of other organisms present.

After overnight incubation the plates were read according to a five grade scale (tables 1 and 2). The grading scales were calibrated by inoculating the media with different dilutions of pure single organism broth cultures of known viable count, handled identically to sputum.

The reproducibility of the technique was studied, using sputum from 31 patients with bronchiectasis and daily purulent sputum, who had been admitted to hospital between exacerbations of chest symptoms. Four hour morning sputum specimens were collected on consecutive days from all 31 patients and a random specimen was also collected from 14 of the patients. Patients were asked not to change their routine practice of postural drainage and breathing exercises, and their medications were not changed. None was receiving antibiotics. A protocol was devised to test the reproducibility of the technique within sputum samples and its variability on serial sputum specimens from the same patient.

\section{BACTERIA ISOLATED FROM THE SPUTUM OF 31 PATIENTS PRODUCING PURULENT SPUTUM DAILY}

The number of patients with Haemophilus spp, Ps aeruginosa, and $S$ aureus isolated from the morning specimens (mean 2.8/patient) and the maximum colonising microbial load grade were recorded.

\section{REPRODUCIBILITY OF THE TECHNIQUE}

DETERMINING COLONISING MICROBIAL LOAD

(i) Within morning sputum specimens processed by a single technician

Twenty three pairs of colonising microbial load plates (22 patients) were prepared and read by the same technician to determine the variability of culture results within each sputum. Each pair of plates was prepared from a single morning sputum specimen.

\section{(ii) Between technicians in the processing of single morning sputum specimens}

Seventeen specimens of morning sputum after homogenisation (12 patients) were processed separately by two technicians and finally read by a single technician.

Twenty two sets of plates ( 22 patients) prepared by 
Table 1 Calibration scales of colonising microbial load ( $S$ aureus and $P$ aeruginosa)

\begin{tabular}{|c|c|c|c|}
\hline \multirow{2}{*}{$\begin{array}{l}\text { Colonising } \\
\text { microbial } \\
\text { load grade }\end{array}$} & \multirow[b]{2}{*}{ Description } & \multicolumn{2}{|c|}{ Midpoint (range) of colony count on log scale ( $\mathrm{No} / \mathrm{ml}$ ) } \\
\hline & & Staphylococcus & Pseudomonas \\
\hline 0 & No growth & - & - \\
\hline \pm & A few isolated colonies & $2.4(0.7-9.4) \times 10^{3}$ & $2(1 \cdot 6-2 \cdot 4) \times 10^{3}$ \\
\hline+ & Light to heavy growth over first two quarters of the plate only & $1.4(0.07-28) \times 10^{5}$ & $3 \cdot 2(0 \cdot 6-16) \times 10^{4}$ \\
\hline++ & $\begin{array}{l}\text { Heavy growth in the first two quarters with growth in the } \\
\text { third quarter of the plate }\end{array}$ & $5(0 \cdot 7-28) \times 10^{6}$ & $5.6(1 \cdot 8-18) \times 10^{5}$ \\
\hline+++ & $\begin{array}{l}\text { Heavy growth in the first two quarters with growth in the } \\
\text { third and fourth quarters of the plate }\end{array}$ & $3(0 \cdot 8-11)) \times 10^{7}$ & $1 \cdot 2(0 \cdot 16-9 \cdot 2) \times 10^{7}$ \\
\hline
\end{tabular}

Table 2 Calibration scales of colonising microbial load ( $H$ influenzae and $H$ parainfluenzae)

\begin{tabular}{lll}
\hline Grade & $\begin{array}{l}\text { Description of selective } \\
\text { growth around the V disc }\end{array}$ & $\begin{array}{l}\text { Midpoint (range) of } \\
\text { colony count on log } \\
\text { scale }(\mathrm{No} / \mathrm{ml})\end{array}$ \\
\hline 0 & No growth & $1.4(0 \cdot 1-24) \times 10^{4}$ \\
\pm & A few colonies & $8.4(0.4-140) \times 10^{4}$ \\
+ & A definite ring of colonies & $4(0.4-34) \times 10^{5}$ \\
++ & A moderate sized ring of colonies & $2.6(0 \cdot 2-36) \times 10^{6}$ \\
+++ & A large ring of colonies & $3.2(0.4-19) \times 10^{7}$ \\
\hline
\end{tabular}

one technician were read separately by two technicians.

VARIABILITY OF THE TECHNIQUE DETERMINING COLINISING MICROBIAL LOAD BETWEEN SERIAL SPUTUM SPECIMENS

(i) Between morning sputum specimens

The colonising microbial loads of 23 morning sputum specimens ( 22 patients) were compared with those of 23 sputum specimens obtained 48 hours later from the same patients and handled identically.

(ii) Between morning and random sputum specimens Fourteen random sputum specimens (14 patients) were collected. The colonising microbial load of these sputum specimens was compared with that of morning sputum from the same patients collected 40-48 hours after the random specimen and handled identically.

\section{VARIABILITY OF MORNING SPUTUM VOLUME AND CHARACTER}

Two morning sputum specimens collected 48 hours apart from each patient were assessed for volume (24 patients), percentage purulence of the whole specimen (27 patients), colour (green, yellow), and intensity of colour of the purulent part (23 patients). Purulence was recorded on a four grade scale: $0-25 \%, 26-50 \%$, $51-75 \%, 76-100 \%$, and intensity of colour was also graded $(+,++,+++)$.
PILOT STUDY OF THIS TECHNIQUE USED TO MONITOR COLONISING MICROBIAL LOAD IN PATIENTS WITH CHRONIC BRONCHIAL SEPSIS DURING TREATMENT WITH ANTIBIOTICS

(i) Intermittent treatment of exacerbations

Twenty five patients (aged 23 to 57 years; 9 men, 16 women) with bronchiectasis and persistent purulent sputum were treated for one year with seven day courses of $3 \mathrm{~g}$ oral amoxycillin twice daily before food, for exacerbations of their chest symptoms, as judged by the patient noticing increasing volume or deepening of the colour of the sputum (mean 14.5 courses/patient, range six to 25 ).

\section{(ii) Continuous oral treatment}

Eleven patients (aged 21 to 61 years; four men, seven women) with bronchiectasis and persistent purulent sputum were treated with $\mathbf{3} \mathrm{g}$ oral amoxycillin twice daily before food for a total of six months.

\section{(iii) Continuous nebulised treatment}

Ten patients (aged 19 to 59 years; three men, seven women) with bronchiectasis and persistent purulent sputum were treated with $1 \mathrm{~g}$ nebulised amoxycillin (diluted in $3.5 \mathrm{ml}$ sterile water) twice daily for a total of six months.

Sputum purulence and colonising microbial load were estimated monthly in the continuously treated groups, but in the intermittently treated group they were measured before and after treatment of the first exacerbation, at the beginning of the second exacerbation, and at intervals of three months. All sputum examinations were performed "blind" by technicians unacquainted with the origin of the samples.

\section{Results}

OCCURRENCE OF ORGANISMS IN SPUTUM

Haemophilus species were isloated from the sputum of 27 of $31(1 \pm, 10+, 11++, 5+++)$ patients; Ps 
aeruginosa from 14 of $31(2 \pm, 4+, 5++$ and $3+++) ; S$ aureus from six of $32(1 \pm, 3+, 1++$ and $1+++$ ); and Streptococcus pneumoniae from one of 31 (colonising microbial load not measured). No organisms were grown from the sputum of two patients.

\section{REPRODUCIBILITY OF THE TECHNIQUE}

(i) Variability within sputum specimens (same technician)

There was no difference in colonising microbial load between 24 of the 32 organisms cultured, a difference of one grade for 11 of 32 and of two grades for only one of 32 . The mean difference was 0.3 grades. The $95 \%$ confidence limits for a difference of up to one grade (31 of 32 ) were $79-100 \%$.

\section{(ii) Variability between technicians processing sputum specimens}

There was no difference in colonising microbial load between 18 of 31 organisms cultured and a difference of one grade for 13 of 31 . The mean difference was 0.4 grades. The $95 \%$ confidence limits for a difference of up to one grade ( 31 of 31 ) were $89-100 \%$.

\section{Variability between technicians reading colonising microbial loads from sputum specimens prepared by a single technician}

There was no difference in colonising microbial load between 39 of 43 organisms cultured and a difference of one grade for only four of 43 . The mean difference was 0.1 grades. The $95 \%$ confidence limits for a difference of up to one grade (43 of 43) were $92-100 \%$.

\section{VARIABILITY BETWEEN SERIAL SPUTUM SPECIMENS COLLECTED 48 HOURS APART FROM THE SAME PATIENT}

\section{(i) Between morning specimens}

There was no difference in colonising microbial load for eight of 38 organisms cultured, a difference of one grade for 14 of 38 , of two grades for 11 of 38 , and of three or more grades for five of 38 . The mean difference was 1.4 grades. The $95 \%$ confidence limits for a difference of up to one grade ( 22 of 38 ) were $41-74 \%$, and for a difference of up to two grades (33 of 38$), 72-96 \%$. Of the 30 differences in colonising microbial load grade, 19 were increases in grade and 11 decreases after the $\mathbf{4 8}$ hours. Different colonising microbial load grades and the different organisms were subject to similar variation.

\section{(ii) Between morning and random specimens}

There was no difference in colonising microbial load for two of 22 organisms cultured, a difference of one grade for 10 of 22 and of two or more grades for nine of 22 . The mean difference was 1.5 grades. The $95 \%$ confidence limits for a difference of up to one grade (12 of 22 ) were $32-76 \%$.

\section{VARIABILITY OF MORNING SPUTUM VOLUME AND CHARACTER}

The initial morning sputum volume ranged from $5-50 \mathrm{ml}$ (median $15 \mathrm{ml}$ ). The mean change (increase or decrease) in volume was by $44 \%$ of the original volume (range 0-114\%). Purulence (\%) initially ranged from $25 \%$ to $100 \%$ (median $50-75 \%$ ). There was no difference in grade after 48 hours in 17 of 27 patients, a difference of one grade in nine of 27 , and by more than one grade in one of 27 . The mean change in grade was 0.4 grades. The $95 \%$ confidence limits for a difference of up to one grade (26 of 27) were $81-100 \%$. The colour of the purulent portion did not change in 22 patients (green in 21 and yellow in one). The other patient's sputum colour changed from a mixture of yellow-green to green. The intensity of colour ranged from + to +++ initially, did not change in 18 of 23 patients, changed by one grade in four of 23 , and by more in one of 23 . The mean change was 0.2 grades. The $95 \%$ confidence limits for a difference of up to one grade (22 of 23) were $78-100 \%$. Sputum volume $(p>0 \cdot 25)$ and purulence $(p>0 \cdot 1)$ did not significantly improve or worsen after the 48 hours and the observed changes did not correlate with the difference in colonising microbial load grade.

PILOT STUDY OF COLONISING MICROBIAL LOAD DETERMINATION DURING TREATMENT OF

CHRONIC BRONCHIAL SEPSIS

(i) Intermittent treatment of exacerbations

An initial high colonising microbial load of $\mathrm{Hae}$ mophilus spp before treatment was reduced in 18 of 25 patients after one week of treatment $(+++$ to ++$)$, but all patients had increased to pre-treatment colonising microbial load by the second exacerbation (fig 1). The colonising microbial loads of Haemophilus spp (grade ++ ), Ps aeruginosa (grade +++ ), and sputum purulence measured at intervals of three months' exacerbations remained constant at the postexacerbation level (fig 1).

\section{(ii) Continuous oral treatment}

There was a gradual reduction in colonising microbial load of both Haemophilus spp $(+++$ to \pm , or 0$)$ and Ps aeruginosa $(+++$ to,+ \pm or 0$)$ in seven of 11 patients who remained in the study after a month (fig 2). In the four of 11 patients who failed to respond clinically and stopped treatment after one month the colonising microbial load had not changed. Sputum purulence was reduced in parallel with colonising microbial load during treatment. 


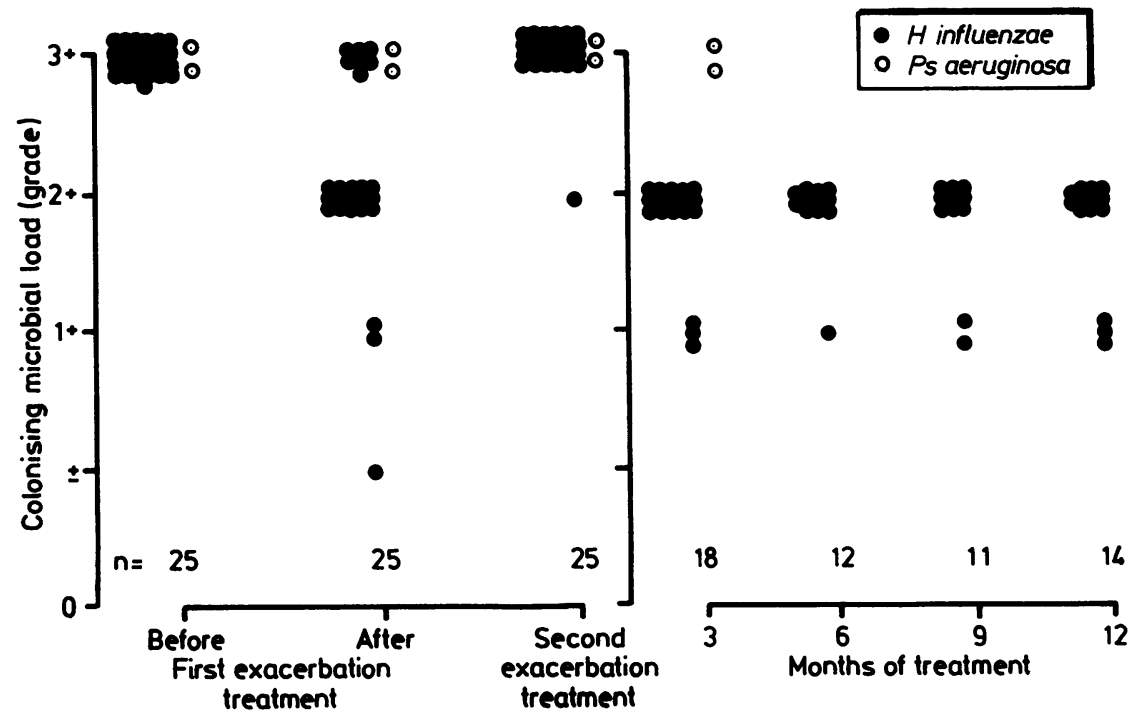

Fig 1 Colonising microbial load of Haemophilus spp and Pseudomonas aeruginosa during treatment for one year with intermittent seven day courses of oral amoxycillin ( $3 \mathrm{~g}$ twice daily) for exacerbations of bronchiectasis.

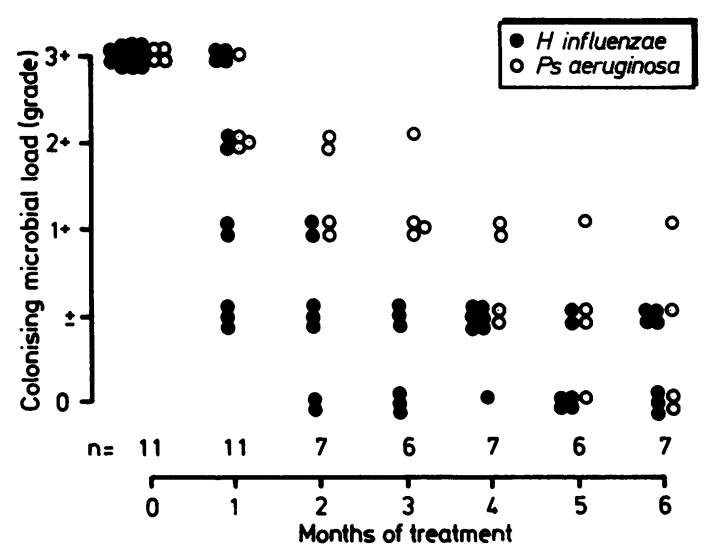

Fig 2 Colonising microbial load of Haemophilus spp and Pseudomonas aeruginosa during treatment of patients with bronchiectasis continuously for six months with oral amoxycillin ( $3 \mathrm{~g}$ twice daily).

(iii) Continuous nebulised treatment

Results were similar to those seen with continuous oral treatment (fig 3). The colonising microbial load of Haemophilus spp reduced from +++ to \pm or 0 and $P$ s aeruginosa from +++ to + or \pm in the eight of 10 patients continuing to be treated after one month. Sputum purulence was again concomitantly reduced.

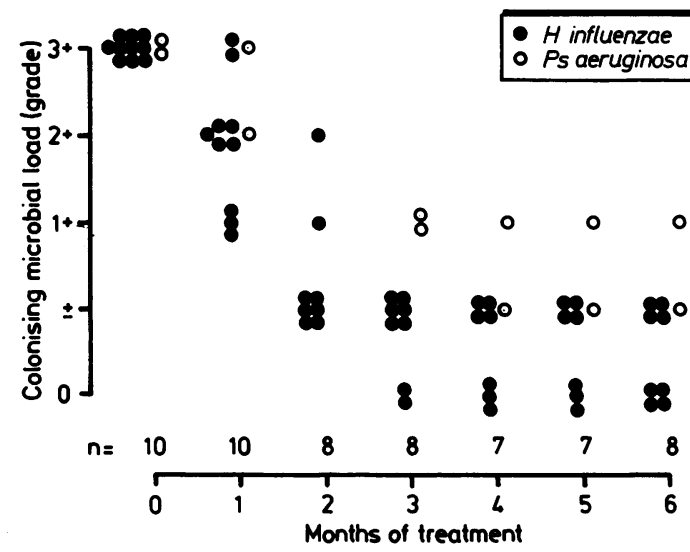

Fig 3 Colonising microbial load of Haemophilus spp and Pseudomonas aeruginosa during treatment of patients with bronchiectasis continuously for six months with nebulised amoxycillin (1 $\mathrm{g}$ twice daily).

\section{Discussion}

Many methods for handling sputum to improve stan- $\stackrel{\mathbb{D}}{\rightarrow}$ dard qualitative culture techniques have been 0 described. A perfect method would identify and 0 quantitate all the important organisms present in the $\stackrel{\mathbb{D}}{\mathbb{D}}$ bronchial tree from a single sample of sputum and at $\vec{\nabla}$ the same time not grow oropharyngeal contaminants 
and commensals.

May elegantly showed the non-uniform distribution of bacteria in a single sputum sample. ${ }^{4} \mathrm{He}$ proposed multiple cultures from each sample, or effective homogenisation of a single sample before culture to increase the number of species isolated. Homogenisation can be achieved by chemicals or enzymes (such as $\mathrm{N}$-acetylcysteine ${ }^{5}$ or pancreatin ${ }^{6}$ ) or mechanical methods, as in this study. Chemical and enzymatic methods, however, are more likely to kill micro-organisms destined for culture. Oropharyngeal contamination of expectorated sputum has been clearly shown. ${ }^{7-9}$ The number of contaminating organisms is usually less than $10^{4} / \mathrm{ml}^{10}$ and seldom greater than $10^{7} / \mathrm{ml}^{11}$ Modifications have been advised at all stages in handling sputum to reduce oropharyngeal contamination: the mouth may be rinsed with water before expectoration ${ }^{11}{ }^{12}$; the sputum washed in vitro with saline ${ }^{13}$ or running tap water'; only purulent areas of sputum should be selected for culture ${ }^{8}$ or the sputum diluted before being plated out for culture. ${ }^{121415} \mathrm{~A}$ standard bacteriological approach to sputum culture with quantitation of organisms has not been introduced despite the variety of methods proposed for homogenisation and the reduction of oropharyngeal contaminationmainly because there are doubts about the validity of these methods. For example, routine dilution of sputum before culture might well reduce the number of pathogens to undetectable levels in some cases. ${ }^{16}$ The rationale behind the practice of washing sputum is the assumption that all the liquid (non-viscous) portion of expectorated sputum is saliva, but a proportion of this liquid may originate from the damaged bronchial tree and thus carry important micro-organisms, which might not be isolated if the sputum were washed.

This study has achieved minimal variability within sputum samples by collecting them over a four hour period and mechanical homogenisation. Our selective culture methods avoid the problem of bacterial overgrowth by upper and lower respiratory tract commensals or coexisting pathogens, apart from the four selected bacterial species. The method also maximises the yield of the four bacteria $H$ influenzae, $H$ parainfluenzae, Ps aeruginosa, and $S$ aureus by the use of selective culture media-selecting according to growth factor requirement and fermenting abilityand allows the rapid identification and quantitation of these bacteria. Haemophilus spp, the main organisms isolated in the sputum during our study, are seldom isolated from the oropharynx without coincidental isolation from the bronchi (direct sampling using double lumen device) in patients with daily mucopurulent sputum. ${ }^{78}$ These four bacteria are those which, in our experience, are most com- monly associated with chronic bronchial sepsis. A study of sputum culture in children with cystic fibrosis ${ }^{15}$ also found that selective culture media are valuable in the isolation and quantitation of the same bacteria. Subcultures were still necessary, however, to distinguish Haemophilus spp from other Gram stain negative bacilli, and serial dilution of sputum was required for quantitation. Using a different culture method for Haemophilus spp $^{2}$ and a reproducible grading system for quantitation of bacteria, our technique avoids time consuming subculture, colony counting, and serial dilution of sputum. The selective culture method for Haemophilus spp has been reported to increase the rate of isolation from five to $80 \%$ in patients with bronchiectasis or adult cystic fibrosis or both. ${ }^{2}$ The selective medium ${ }^{2}$ has been modified by the addition of $\mathrm{N}$-acetyl-D-glucosamine to encourage growth of $H$ influenzae spheroplasts, ${ }^{17}$ by minor reductions in the concentrations of bacitracin and haemin, and by the addition of supplementary glucose. Occasionally, subculture is required to check the identity of a dubious organism.

The comparison of morning sputums collected 48 hours apart showed that, despite collection over four hours and minimal variability due to technique, there is considerable variation in the bacterial flora of the expectorated sputum. This inherent variation is similar to that seen between random and morning sputum specimens. There was minimal change in the character of the morning specimens and the pronounced changes in volume did not correlate with the changes in colonising microbial load. Two unexplored possibilities, which may explain the inherent sputum variability, are remaining differences in the site of origin of the sputum despite four hour collections and variations in the length of time between first colonisation of airway mucus by micro-organisms and expectoration. Multiple specimens may reduce inherent variability, but they are very inconvenient and were not included in our pilot study.

Our new technique has enabled objective assessment of the effect of different antibiotic regimens on the level of colonisation of the chronically infected respiratory tract. This has shown that, even in a dose chosen to achieve a bactericidal concentration in the sputum of such patients $(3 \mathrm{~g}$ oral amoxycillin twice daily before food), solely treating exacerbations of bronchiectasis with antibiotics only temporarily reduces the level of colonisation of the antibiotic sensitive organism and does not affect sputum volume or purulence. In contrast, prolonged doses of oral or nebulised antibiotic result in considerable and progressive reduction in colonisation with both antibiotic sensitive Haemophilus spp and antibiotic resistant Ps aeruginosa over the period of treatment. That this coincides with reduction in sputum puru- 
lence suggests that the host mediated inflammatory response to the colonising organisms diminishes with treatment. As both products of $H$ influenzae and $H$ parainfluenzae ${ }^{18}$ and also serine-proteinase from neutrophils ${ }^{19}$ have been shown to slow human ciliary beating in vitro, reduction in the numbers of these bacteria and the inflammation they provoke may permit improvement in mucociliary clearance. Hence the apparent paradox of the reduction in the numbers of antibiotic resistant $P s$ aeruginosa may be explained on the basis of gradual recovery of host clearance mechanisms. Prolonged antibacterial treatment coincided with a decrease in sputum volume and purulence, but it would be unwise to draw conclusions on the clinical sequelae of reduction in colonising microbial load with this treatment as this was an open study (as far as clinical details were concerned). Nevertheless, the results are sufficiently encouraging to justify formal assessment in a double blind study using placebo controls, which is now in progress.

DC was supported by the Chest, Heart and Stroke Association. This work was supported by the Wellcome Trust.

\section{References}

1 Cole PJ. A new look at the pathogenesis and management of persistent bronchial sepsis: a "vicious circle" hypothesis and its logical therapeutic connotations. In: Strategies for the management of chronic bronchial sepsis. Oxford: Medicine Publishing Foundation, 1984:1-20.

2 Roberts DE, Cole P. Use of selective media in bacteriological investigation of patients with chronic suppurative respiratory infection. Lancet 1980;i:796-7.

3 Roberts DE, Higgs E, Prior C, Cole PJ. A simple selective bacteriological medium designed to distinguish Haemophilus influenzae from $H$ parainfluenzae in sputum. Thorax 1985; 40:237.

4 May JR. The bacteriology of chronic bronchitis. Lancet 1953;ii:534-7.

5 Mead GR, Woodhams AW. N-acetyl-L-cysteine as liquefying agent in the bacteriological examination of sputum. Tubercle 1964;45:370-3.
6 Rawlins GA. Liquefaction of sputum for bacteriological examination. Lancet 1953;ii:538-9.

7 Brumfitt W, Willoughby MLN, Bromley LL. An evaluation of sputum examination in chronic bronchitis. Lancet 1957;ii: $\hat{\sigma}$ 1306-9.

8 Lees AW, McNaught W. Bacteriology of lower respiratory tract secretions in "normals" and chronic bronchitics. Lancet 1959; ii:1112-5.

9 Bartlett JG, Finegold SM. Bacteriology of expectorated sputum with quantitative culture and wash technique compared to transtracheal aspirates. Am Rev Respir Dis 1978;117:1019-27.

10 Dixon JMS, Miller DC. Value of dilute inocula in cultural examination of sputum. Lancet 1965 ;ii:1046-8.

11 Monroe PW, Muchmore HG, Felton FG, Pirtle JK. Quantitation of micro-organisms in sputum. Appl Microbiol 1969;18:214-20.

12 Pirtle JK, Monroe PW, Smalley TK. Mohr JA, Rhoades ER. Diagnostic and therapeutic advantages of serial quantitative cultures of fresh sputum in acute bacterial pneumonia. Am Rev Respir Dis 1969;100:831-8.

13 Lapinski EM, Flakas ED, Taylor BC. An evaluation of some methods for culturing sputum from patients with bronchitis and emphysema. Am Rev Respir Dis 1964;89:760-3.

14 Kilbourn JP, Campbell RA, Grach JL, Willis MD. Quantitative bacteriology of sputum. Am Rev Respir Dis 1968;98:810-8.

15 Wong K, Roberts MC, Owens L, Fife M, Smith AL. Selective media for the quantitation of bacteria in cystic fibrosis sputum. Med Microbiol 1984;17:113-9.

16 MacCulloch D, Prairie J. A comparison of direct and diluted sputum culture results. $N Z$ Med J 1973;78:442-3.

17 Roberts D, Higgs E, Rutman A, Cole P. Isolation of spheroplastic forms of Haemophilus influenzae from sputum in conventionally treated chronic bronchial sepsis using selective medium supplemented with $\mathrm{N}$-acetyl-D-glucosamine: a possible reservoir for re-emergence of infection. $\mathrm{Br}$ Med $J$ 1984; 289:1409-12.

18 Wilson R, Pitt T, Rutman A, Roberts D, Cole P. Haemophilus influenzae and Haemophilus parainfluenzae slow and disorganise the beating of human cilia in vitro. Clin Sci 1986; 70(suppl):26.

19 Smallman LA, Hill SL, Stockley RA. Reduction of ciliary beat frequency in vitro by sputum from patients with bronchiectasis: a serine proteinase effect. Thorax 1984;39:663-7.

Requests for reprints to: Dr PJ Cole, Host Defence Unit, Department of Thoracic Medicine, Cardiothoracic Institute, Brompton Hospital, Fulham Road, London SW3 6HP, England. 